\title{
Thoughts on Thermomechanics
}

\author{
Walter Noll \\ Department of Mathematical Sciences \\ Carnegie Mellon University, Pittsburgh, PA 15238, USA
}

\begin{abstract}
First, I give a short description of the history of the interpretation of the second law of thermodynamics as a restriction on the kind of material properties that physical systems occurring in nature can have. Second, I describe the desirability of developing a framework, using the concept of a state of a material element, for developing a general thermomechanical theory of simple materials. Third, I give a taste of the mathematical infrastructure needed for such a theory. Finally, I give an outline of the paper [NS] by Brian Seguin and myself which may be a model for the first few chapters of future textbooks on continuum thermomechanics.
\end{abstract}

Key Words: Thermomechanics, Second Law, Simple materials, mathematical infrastructure.

\section{History and Outlook}

Until about 50 years ago, the overlap between thermodynamics and mechanics was very limited. It dealt mostly with the laws relating thermal energy and mechanical energy. However, the first and second laws of thermodynamics were not connected with continuum mechanics. Courses in thermodynamics, elasticity, and hydrodynamics had very little connection. The very term "thermomechanics" had not been invented. Even now the term is still not in the dictionary. Yet there are books with this title, and typing this term into Google yields 65000 hits.

Of course, the development of statistical mechanics in the 19th century yielded an important connection between particle mechanics and thermodynamics, but this did not seem to have much relevance to continuum mechanics.

When working on The Non-Linear Field Theories of Mechanics [NLFT] with Clifford Truesdell in the early 1960's, I encountered the problem of justifying the formula relating the stress in elasticity to the gradient of the internal energy with respect to the transplacement gradient ${ }^{1}$. First, I naively conjectured that this formula can be derived from the law of conservation of energy. It cannot. What is needed is an appropriate interpretation of the second law of thermodynamics.

Here is the interpretation:

Dissipation principle: For all thermodynamic processes that are admissible for a given constitutive assumption, the entropy production must be positive or zero.

The decisive word in this postulate is the quantifier all. It makes the postulate a restrictive con- dition on the constitutive assumptions that can be imposed on systems of the type under consideration. Indeed, if constitutive assumptions are laid down at will and without restriction, the entropy production can be expected to be positive or zero only for some but not for all admissible processes. Thus, the second law is not a restriction on the kind of processes that can occur in nature, but a restriction on the kind of material properties that physical systems occurring in nature can have. This is not made clear in most explanations of the second law in the textbook literature on thermodynamics. An exception is the Lectures on Physics by R. Feynman. He writes: "So we see that a substance's properties must be limited in a certain way; one cannot make up anything he wants .... This principle, this limitation, is the only real rule that comes out of thermodynamics." (See pp. 44-6 and 44-7 of reference [F].)

What happened next is described by the following excerpt from Bernard Coleman's Memories of Clifford Truesdell $[\mathrm{C}]$ :

"In the early 1960's Walter Noll put to me the idea, as if it should be obvious to everyone, that the inequality is a restriction on all processes that are admissible in a material of which the body is composed, and, because one defines each material by giving a set of constitutive relations, the Clausius-Duhem inequality, as it must hold for all processes compatible with these relations, becomes a restriction on constitutive relations. In another act of great generosity, Walter suggested that we develop the idea together. It took a while to sort the argument out and to present it in a way that would convince the wary."

The result of our efforts was the paper The

\footnotetext{
${ }^{1}$ In 1958, I introduced the unfortunate terms "configuration" and "deformation" for what are now called "placement" and "transplacement". I apologize. Since these old terms were used in [NLFT], they were widely accepted, and I am now in the ironic position to fight against something that I started.
} 
Thermodynamics of Elastic Materials with Heat Conduction and Viscosity [CN]. There we gave rigorous mathematical proofs that the second law implies, among other things, that the heat conduction coefficients and the viscosities in fluids must be positive or zero. This paper became a citation classic, and the procedure used in this paper has been dubbed the "Coleman-Noll procedure". Typing this term into Google yields about 3500 hits.

In The Non-Linear Field Theories of Mechanics, published in 1965, Clifford Truesdell and I presented what we called "The general theory of material behavior" (Chapter C of [NLFT]). About seven years later, in about 1971, I realized that this "general theory" cannot be the final word. (See the Preface to the Second Edition, published in 1992.) What was needed is a general framework for the formulation of constitutive laws, using the concept of a state of a material element. I presented such a framework in the paper A New Mathematical Theory of Simple Materials, 50 pages long, published in 1972 [N1]. This framework dealt only with purely mechanical phenomena. However, in the end (Sect.21), I proposed that this framework should be generalized to become a general thermomechanical theory. It took 35 years before somebody started to develop such a generalization. It was my doctoral student Brian Seguin, now at the end of his fourth year as a graduate student at CMU. His work could be of groundbreaking significance.

I was disappointed that my 1972 paper [N1] mentioned above generated very little resonance. The reason is that it required familiarity with a mathematical infrastructure that was and still is foreign to most people interested in thermomechanics. Here is an example: A letter from the editor of the Reviews of Modern Physics, written in 1988, informed me that a 1961 paper by Bernard Coleman and me had become a citation classic and that they would welcome receiving other papers from me. In 1995 I submitted a paper entitled On Material Frame Indifference to this journal. I thought that this paper should be of interest to an audience wider than just those interested only in the mathematics of continuum physics. However, my paper was rejected even though the editor conceded that "the article is clearly written". Here are some quotes from the reviewer:

"I enjoyed reading this paper and very much would like to see it published. I am afraid, however, that the Reviews of Modern Physics is not the appropriate place. I believe that the overwhelming majority of the readers of the journal will consider the paper unreadable. Not because the material presented is intrinsically difficult, but rather because the author's individual form of the 'Bourbakian' style is far removed from anything that physicists are willing to digest. .... Professor Noll is highly respected in the mathematical community and has more than once proved himself to be ahead of his time. ..."

The paper is now part 2 of my Five Contributions to Natural Philosophy [FC].

In a recent exchange of e-mails, another scientist also told me that he has "reservations for the Boubakization of mechanics". Here is my answer to him:

"My more recent work is almost entirely expressed in terms of the stage 3 infrastructure of mathematics. This is not just a matter of notation or style. The language of stage 2 mathematics is simply insufficient to describe my ideas. I am convinced that this stage 3 infrastructure will eventually prevail, even among physicists and engineering scientist, but it may take another 40 years. To call it 'Bourbakization' is a gross mischaracterization."

The stage 2 mathematical infrastructure is based on the concepts of variables, constants, and parameters. The stage 3 mathematical infrastructure is based on the concepts of sets and mappings. For a detailed explanation, see my essay entitled The Conceptual Infrastructure of Mathematics [N2].

One of the issues that has created much confusion in the literature on thermomechanics is the Principle of Material Frame Indifference. It is often confused with material symmetry. I deal with this issue in part 2 of the Five Contributions to Natural Philosophy [FC] mentioned above. The best way to avoid using this Principle and not confuse it with material symmetry is to formulate constitutive laws without using a frame of reference. This has been done in [N1], [FC], [N3], and also in Brian Seguin's work to be described in a later presentation.

I am tired of being ahead of my time and hope to induce some people to become familiar with some of the stage 3 mathematical infrastructure that will be needed, for example, to understand the paper Basic Concepts of Thermomechanics [NS1] by Brian Seguin and me outlined in Section 3 below. A detailed treatment of some of this infrastructure is presented in my textbook [FDS], now available free of charge on the internet. The following gives a taste of it.

\section{Mathematical infrastructure}

\subsection{Sets and Mappings}

The set of real numbers will be denoted by $\mathbb{R}$. The set of positive numbers (including zero) will be denoted by $\mathbb{P}$ while the set of strictly positive numbers (excluding zero) will be denoted by $\mathbb{P}^{\times}$. 
In order to specify a mapping $f: \mathcal{A} \longrightarrow \mathcal{B}$, one first has to prescribe two sets, $\mathcal{A}$ and $\mathcal{B}$, and then a definite procedure, called the evaluation rule of $f$, which assigns to each element $a \in \mathcal{A}$ exactly one element $f(a) \in \mathcal{B}$. The set $\mathcal{A}$ is called the domain of $f$ and the set $\mathcal{B}$ is called the codomain of $f$. We say that $f$ is a mapping from $\mathcal{A}$ to $\mathcal{B}$ or maps $\mathcal{A}$ to $\mathcal{B}$.

For every set $\mathcal{A}$ we have the identity mapping $1_{\mathcal{A}}: \mathcal{A} \longrightarrow \mathcal{A}$ of $\mathcal{A}$, defined by $1_{\mathcal{A}}(a):=a$ for all $a \in \mathcal{A}$.

The composite $g \circ f: \mathcal{C} \longrightarrow \mathcal{A}$ of two mappings $f: \mathcal{A} \longrightarrow \mathcal{B}$ and $g: \mathcal{B} \longrightarrow \mathcal{C}$ is defined by

$$
(g \circ f)(a):=g(f(a)) \text { for all } a \in \mathcal{A} \text {. }
$$

Now let a mapping $f: \mathcal{A} \longrightarrow \mathcal{B}$ be given. We say that $f$ is injective if, for every $b \in \mathcal{B}$, there is at most one $a \in \mathcal{A}$ such that $f(a)=b$. We say that $f$ is surjective if, for every $b \in \mathcal{B}$, there is at least one $a \in \mathcal{A}$ such that $f(a)=b$. The mapping $f$ is both injective and surjective if and only if, for every $b \in \mathcal{B}$, there is exactly one $a \in \mathcal{A}$ such that $f(a)=b$. In that case, we say that $f$ is invertible and we define the inverse $f^{\leftarrow}: \mathcal{B} \longrightarrow \mathcal{A}$ by the procedure which associates with each $b \in \mathcal{B}$ the only $a:=f^{\leftarrow}(b) \in \mathcal{A}$ which satisfies $f(a)=b$. We then have

$$
f \circ f^{\leftarrow}=1_{\mathcal{B}} \quad \text { and } \quad f^{\leftarrow \circ f=1_{\mathcal{A}} .}
$$

The set

$$
\text { Rng } f:=\{f(a) \in \mathcal{B} \mid a \in \mathcal{A}\}
$$

is called the range of $f$. We have $\operatorname{Rng} f=\mathcal{B}$ if and only if $f$ is surjective.

The mapping $f$ induces a mapping $f_{>}: \operatorname{Sub} \mathcal{A} \longrightarrow \operatorname{Sub} \mathcal{B}$, from the set $\operatorname{Sub} \mathcal{A}$ of all subsets of $\mathcal{A}$ to the set Sub $\mathcal{B}$ of all subsets of $\mathcal{B}$. It is defined by

$$
f_{>}(A):=\{f(a) \in \mathcal{B} \mid a \in \mathcal{A}\} \quad \text { for all } A \in \operatorname{Sub} \mathcal{A}
$$

and called the image mapping of $f$.

Given two sets, $\mathcal{A}_{1}$ and $\mathcal{A}_{2}$, one can form the set-product $\mathcal{A}_{1} \times \mathcal{A}_{2}$ of $\mathcal{A}_{1}$ and $\mathcal{A}_{2}$. It is defined by

$$
\mathcal{A}_{1} \times \mathcal{A}_{2}:=\left\{\left(a_{1}, a_{2}\right) \mid a_{1} \in \mathcal{A}_{1}, a_{2} \in \mathcal{A}_{2}\right\} .
$$

Given $h_{1}: \mathcal{D} \longrightarrow \mathcal{A}_{1}$ and $h_{2}: \mathcal{D} \longrightarrow \mathcal{A}_{2}$ one can construct the term-wise evaluation mapping $\left(h_{1}, h_{2}\right): \mathcal{D} \longrightarrow \mathcal{A}_{1} \times \mathcal{A}_{2}$ by

$$
\left(h_{1}, h_{2}\right)(d):=\left(h_{1}(d), h_{2}(d)\right) \text { for all } d \in \mathcal{D} .
$$

Conversely, given $h: \mathcal{D} \longrightarrow \mathcal{A}_{1} \times \mathcal{A}_{2}$ then there are mappings $h_{1}: \mathcal{D} \longrightarrow \mathcal{A}_{1}$ and $h_{2}: \mathcal{D} \longrightarrow \mathcal{A}_{2}$ such

\footnotetext{
${ }^{2}$ For a matrix-free definition see Sect.14 of [FDS], Vol.II
}

that $h=\left(h_{1}, h_{2}\right)$. The mappings $h_{1}$ and $h_{2}$ are called the component mappings of $h$. A similar result holds when the codomain of $h$ is the product of more the two sets.

\subsection{Linear Algebra}

Here we deal only with finite-dimensional real linear spaces. Let $\mathcal{T}_{1}$ and $\mathcal{T}_{2}$ be such linear spaces. We use the notation $\operatorname{Lin}\left(\mathcal{T}_{1}, \mathcal{T}_{2}\right)$ for the set of all linear mappings from $\mathcal{T}_{1}$ to $\mathcal{T}_{2}$. This set also has the structure of a linear space and $\operatorname{dim} \operatorname{Lin}\left(\mathcal{T}_{1}, \mathcal{T}_{2}\right)=$ $\operatorname{dim} \mathcal{T}_{1} \times \operatorname{dim} \mathcal{T}_{2}$. Given $\mathbf{L} \in \operatorname{Lin}\left(\mathcal{T}_{1}, \mathcal{T}_{2}\right)$ and $\mathbf{v} \in \mathcal{T}_{1}$ we denote by $\mathbf{L v}$ the element of $\mathcal{T}_{2}$ that $\mathbf{L}$ assigns to v.

If $\mathbf{L}_{1}$ and $\mathbf{L}_{2}$ are both linear mappings such that the composite $\mathbf{L}_{1} \circ \mathbf{L}_{2}$ is meaningful then we will denote this composite simply by $\mathbf{L}_{1} \mathbf{L}_{2}$. If a linear mapping $\mathbf{L}$ is invertible, we denote its inverse by $\mathbf{L}^{-1}$. We denote by $\operatorname{Lis}\left(\mathcal{T}_{1}, \mathcal{T}_{2}\right)$ the set of all invertible linear mappings, i.e. linear isomorphisms, from $\mathcal{T}_{1}$ to $\mathcal{T}_{2}$. This set is non-empty if and only if $\operatorname{dim} \mathcal{T}_{1}=\operatorname{dim} \mathcal{T}_{2}$. We use the abbreviations

$$
\operatorname{Lin} \mathcal{T}:=\operatorname{Lin}(\mathcal{T}, \mathcal{T}) \text { and } \operatorname{Lis} \mathcal{T}:=\operatorname{Lis}(\mathcal{T}, \mathcal{T}) .
$$

The second of these sets forms a group with respect to composition, called the linear group of $\mathcal{T}$.

The dual of a linear space $\mathcal{T}$ is defined by

$$
\mathcal{T}^{*}:=\operatorname{Lin}(\mathcal{T}, \mathbb{R}) .
$$

In accordance with the general rule of denoting the evaluation of linear mappings, the value of $\boldsymbol{\lambda} \in \mathcal{T}^{*}$ at $\mathbf{v} \in \mathcal{T}$ will be be denoted simply by $\boldsymbol{\lambda} \mathbf{v}$. The dual $\mathcal{T}^{* *}$ of the dual space $\mathcal{T}^{*}$ will be identified with $\mathcal{T}$ in such a way that the value at $\boldsymbol{\lambda} \in \mathcal{T}^{*}$ of the element of $\mathcal{T}^{* *}$ identified with $\mathbf{v} \in \mathcal{T}$ is $\mathbf{v} \boldsymbol{\lambda}:=\boldsymbol{\lambda} \mathbf{v}$. We have $\operatorname{dim} \mathcal{T}^{*}=\operatorname{dim} \mathcal{T}$.

Given $\mathbf{v} \in \mathcal{T}_{2}$ and $\boldsymbol{\lambda} \in \mathcal{T}_{1}^{*}$ we define the tensor product $\mathbf{v} \otimes \boldsymbol{\lambda} \in \operatorname{Lin}\left(\mathcal{T}_{1}, \mathcal{T}_{2}\right)$ of $\mathbf{v}$ and $\boldsymbol{\lambda}$ by

$$
(\mathbf{v} \otimes \boldsymbol{\lambda}) \mathbf{u}:=(\boldsymbol{\lambda} \mathbf{u}) \mathbf{v} \text { for all } \mathbf{u} \in \mathcal{T}_{1} .
$$

The dual of Lin $\mathcal{T}$ contains a special element $\operatorname{tr} \in(\operatorname{Lin} \mathcal{T})^{*}$ called the trace which is characterized by the property

$$
\operatorname{tr}(\mathbf{v} \otimes \boldsymbol{\lambda})=\boldsymbol{\lambda} \mathbf{v} \quad \text { for all } \mathbf{v} \in \mathcal{T}, \boldsymbol{\lambda} \in \mathcal{T}^{*} .
$$

Another mapping of interest is the determinant det $:$ Lin $\mathcal{T} \longrightarrow \mathbb{R} .^{2}$ We use the notation

$$
\operatorname{Unim} \mathcal{T}:=\{\mathbf{L} \in \operatorname{Lis} \mathcal{V}|| \operatorname{det} \mathbf{L} \mid=1\}
$$

for the unimodular group, which is a subgroup of Lis $\mathcal{T}$, and the notation

$$
\operatorname{Unim}^{+} \mathcal{T}:=\{\mathbf{L} \in \operatorname{Lis} \mathcal{V} \mid \operatorname{det} \mathbf{L}=1\}
$$


for the proper unimodular group, which is a subgroup of Unim $\mathcal{T}$.

To every $\mathbf{L} \in \operatorname{Lin}\left(\mathcal{T}_{1}, \mathcal{T}_{2}\right)$ one can associate exactly one element $\mathbf{L}^{\top} \in \operatorname{Lin}\left(\mathcal{T}_{2}^{*}, \mathcal{T}_{1}^{*}\right)$, called the transpose of $\mathbf{L}$, characterized by the condition that

$$
\boldsymbol{\lambda}(\mathbf{L v})=\left(\mathbf{L}^{\top} \boldsymbol{\lambda}\right) \mathbf{v} \quad \text { for all } \mathbf{v} \in \mathcal{T}_{1}, \boldsymbol{\lambda} \in \mathcal{T}_{2}^{*} .
$$

The space $\operatorname{Lin}\left(\mathcal{T}, \mathcal{T}^{*}\right)$ will be identified with the space of all bilinear forms on $\mathcal{T}$. The subspace

$$
\operatorname{Sym}\left(\mathcal{T}, \mathcal{T}^{*}\right):=\left\{\mathbf{L} \in \operatorname{Lin}\left(\mathcal{T}, \mathcal{T}^{*}\right) \mid \mathbf{L}^{\top}=\mathbf{L}\right\}
$$

of $\operatorname{Lin}\left(\mathcal{T}, \mathcal{T}^{*}\right)$ will be identified with the space of all symmetric bilinear forms. The subset

$$
\begin{array}{r}
\operatorname{Pos}^{+}\left(\mathcal{T}, \mathcal{T}^{*}\right):=\left\{\mathbf{G} \in \operatorname{Sym}\left(\mathcal{T}, \mathcal{T}^{*}\right) \mid(\mathbf{G v}) \mathbf{v}>0\right. \\
\text { for all } \mathbf{v} \in \mathcal{T} \text { with } \mathbf{v} \neq \mathbf{0}\}
\end{array}
$$

of $\operatorname{Sym}\left(\mathcal{T}, \mathcal{T}^{*}\right)$ will be identified with the set of all strictly positive bilinear forms. It is an open subset and a linear cone in $\operatorname{Sym}\left(\mathcal{T}, \mathcal{T}^{*}\right)$, but not a subspace. We note that $\operatorname{Pos}^{+}\left(\mathcal{T}, \mathcal{T}^{*}\right) \subset \operatorname{Lis}\left(\mathcal{T}, \mathcal{T}^{*}\right)$.

A (genuine) inner-product space $\mathcal{V}$ is a linear space endowed with additional structure by singling out a specific element ip $\in \operatorname{Pos}^{+}\left(\mathcal{V}, \mathcal{V}^{*}\right)$, called the inner-product. The inner-product is used to identify the linear space $\mathcal{V}$ with its dual $\mathcal{V}^{*}$. It is customary to use the notation

$$
\mathbf{v} \cdot \mathbf{u}:=(\text { ipv }) \mathbf{u} \text { for all } \mathbf{v}, \mathbf{u} \in \mathcal{V}
$$

The magnitude $|\mathbf{u}|$ of an element $\mathbf{u} \in \mathcal{V}$ is defined by $|\mathbf{u}|:=\sqrt{\mathbf{u} \cdot \mathbf{u}}$.

If $\mathcal{T}$ is just a linear space without inner product then the entire theory of inner-product spaces can be applied relative to any $\mathbf{G} \in \operatorname{Pos}^{+}\left(\mathcal{T}, \mathcal{T}^{*}\right) .^{3}$ For example, for each $\mathbf{G} \in \operatorname{Pos}^{+}\left(\mathcal{T}, \mathcal{T}^{*}\right)$ one can define

$$
\begin{aligned}
& \text { Orth } \mathbf{G}:=\left\{\mathbf{A} \in \operatorname{Lis} \mathcal{T} \mid \mathbf{A}^{\top} \mathbf{G} \mathbf{A}=\mathbf{G}\right\} \\
& \text { Orth }^{+} \mathbf{G}:=\{\mathbf{A} \in \text { Orth } \mathbf{G} \mid \operatorname{det} \mathbf{A}=1\} .
\end{aligned}
$$

The first of these is the orthogonal group of $\mathbf{G}$, a subgroup of Unim $\mathcal{T}$, and the second of these is the proper orthogonal group of $\mathbf{G}$, a subgroup of Unim $^{+} \mathcal{T}$. The following two facts about orthogonal groups are of interest:

(1) For all $\mathbf{G}_{1}, \mathbf{G}_{2} \in \operatorname{Pos}^{+}\left(\mathcal{T}, \mathcal{T}^{*}\right)$ we have

Orth $\mathbf{G}_{2}=$ Orth $\mathbf{G}_{2} \Longleftrightarrow \mathbf{G}_{1}=c \mathbf{G}_{2}$ for some $c \in \mathbb{P}^{\times}$

(2) The groups Orth $\mathbf{G}$ are all maximal subgroups of Unim $\mathcal{T}{ }^{4}$

In the applications to thermomechanics, a threedimensional linear space $\mathcal{T}$ represents an infinitesimal element of a continuous body. The elements
$\mathbf{G} \in \operatorname{Pos}^{+}\left(\mathcal{T}, \mathcal{T}^{*}\right)$ represent the configurations of the element. A deformation is simple a change of configuration, and a deformation process is a mapping $\mathbf{P}: I \longrightarrow \operatorname{Pos}^{+}\left(\mathcal{T}, \mathcal{T}^{*}\right)$, where $I$ is a non-empty real interval.

\section{Basic Thermomechanics}

\subsection{Description}

Here is an outline of the paper entitled Basic Concepts of Thermomechanics [NS1] by Brian Seguin and me, which is intended to serve as a model for the first few chapters of future textbooks on continuum mechanics and continuum thermomechanics. It differs from most existing such textbooks in several important respects:

1) It uses the stage 3 mathematical infrastructure mentioned above.

2) It is completely coordinate-free and $\mathbb{R}^{n}$-free when dealing with basic concepts.

3) It does not use a fixed physical space. Rather, it employs an infinite variety of frames of reference, each of which is a Euclidean space. The motivation for avoiding physical space can be found in Part 1, entitled On the Illusion of Physical Space, of [FC]. Here, the basic laws are formulated without the use of a physical space or any external frame of reference. The only frames of reference used there are internal ones, generated by the configurations of the body systems used.

4) It considers inertia as only one of many external forces and does not confine itself to using only inertial frames of reference. Hence kinetic energy, which is a potential for inertial forces, does not appear separately in the energy balance equation. In particle mechanics, inertia plays a fundamental role and the subject would collapse if it is neglected. Not so in continuum mechanics, where it is often appropriate to neglect inertia, for example when analyzing the motion of toothpaste when it is extruded slowly from a tube.

\subsection{Physical Systems}

In the mid 1950's I regularly taught courses for engineering students with the titles Statics and Dynamics. In Statics, the students were asked to con. sider some system (a building, a bridge, or a machine), draw free-body diagrams, and apply to each of these the balance of forces and torques. This often gave enough linear equations to determine the stresses in each of the pieces of the system. In $D y$ namics, the students were asked to apply the same procedure as in Statics, except that inertial forces are

\footnotetext{
${ }^{3}$ This insight, to the best of my knowledge, was first employed in [N1].

${ }^{4}$ The proof is given in [N5].
} 
taken into account. This often led to linear differential equations. I then wondered what the underlying conceptual background of all this was. Here is the result:

Definition 1. An ordered set $\Omega$ with order $\prec$ is said to be materially ordered if the following axioms are satisfied:

(MO1) $\Omega$ has a maximum ma and a minimum mn.

(MO2) Every doubleton has an infimum.

(MO3) For every $p \in \Omega$ there is exactly one member of $\Omega$ denoted by $p^{\text {rem }}$, such that inf $\left\{p, p^{\text {rem }}\right\}=\mathrm{mn}$ and $\sup \left\{p, p^{\text {rem }}\right\}=$ ma.

$$
\begin{aligned}
& \text { (MO4) }\left(\inf \left\{p, q^{\mathrm{rem}}\right\}=\mathrm{mn}\right) \\
& \Longrightarrow p \prec q \quad \text { for all } p, q \in M .
\end{aligned}
$$

The mapping rem $:=\left(p \mapsto p^{\mathrm{rem}}\right): \Omega \longrightarrow \Omega$ is called the remainder mapping in $\Omega$.

Here $\Omega$ is considered to consist of the whole system and all of its parts. Given $a, b \in \Omega, a \prec b$ is read " $a$ is a part of $b$ ". The maximum ma is the "material all", i.e. the whole system, and the minimum $\mathrm{mn}$ is the "material nothing". The $\inf \{a, b\}$ is the overlap of $a$ and $b$, and $a^{\text {rem }}$ is the part of the whole system ma that remains after $a$ has been removed. With this in mind, the two conditions (MO3) and (MO4) above are very natural.

Theorem 1: Let $\Omega$ be a materially ordered set. Then $\Omega$ has the structure of a Boolean algebra with

$$
\begin{gathered}
p \wedge q:=\inf \{p, q\} \text { and } p \vee q:=\sup \{p, q\} \\
\text { for all } p, q \in \Omega,
\end{gathered}
$$

The proof is highly non-trivial. The best version is given in [NS2].

\subsection{Additive Mappings a and Interactions}

Let $\Omega$ be a materially ordered set and $\mathcal{W}$ a linear space. We say that the parts $p$ and $q$ are separate if $p \wedge q=\mathrm{mn}$. We use the notation

$$
\left(\Omega^{2}\right)_{\text {sep }}:=\left\{(p, q) \in \Omega^{2} \mid p \wedge q=\mathrm{mn}\right\} .
$$

Definition 2. A function $\mathbf{H}: \Omega \longrightarrow \mathcal{W}$ is said to be additive if

$$
\begin{gathered}
\mathbf{H}(p \vee q)=\mathbf{H}(p)+\mathbf{H}(q) \\
\text { for all }(p, q) \in\left(\Omega^{2}\right)_{\text {sep }} .
\end{gathered}
$$

For every $p \in \Omega$ we put $\Omega_{p}:=\{q \in \Omega \mid q \prec p\}$, which again has the structu of a materially ordered set.

Definition 3. A function $\mathbf{I}:\left(\Omega^{2}\right)_{\text {sep }} \longrightarrow \mathcal{W}$ is said to be an interaction if, for all $p \in \Omega$, both

$\mathbf{I}\left(\cdot, p^{\mathrm{rem}}\right): \Omega_{p} \longrightarrow \mathcal{W}$ and $\mathbf{I}\left(p^{\mathrm{rem}}, \cdot\right): \Omega_{p} \longrightarrow \mathcal{W}$ are additive.

The resultant $\operatorname{Res}_{\mathbf{I}}: \Omega \rightarrow \mathcal{W}$ of a given interaction $I$ is defined by

$$
\operatorname{Res}_{\mathbf{I}}(p):=\mathbf{I}\left(p, p^{\mathrm{rem}}\right) \quad \text { for all } p \in \Omega
$$

We say that a given interaction is skew if

$$
\mathbf{I}(q, p)=-\mathbf{I}(p, q) \text { for all }(p, q) \in\left(\Omega^{2}\right)_{\text {sep }} .
$$

Theorem 2: An interaction is skew if and only if its resultant is additive.

The proof of this result is fairly easy but not entirely trivial.

Remark 1: The concept of an interaction is an abstraction. Its values may have the interpretation of forces, torques, or heat transfers. In most of the past literature these cases were treated separately even though much of the underlying mathematics is the same for all. Thus, this abstraction, like most others, is a labor saving device.

\subsection{Continuous Bodies}

A continuous body system is a set $\mathcal{B}$ of material points, endowed with structure by the specification of a non-empty set $\operatorname{Conf} \mathcal{B}$, whose elements are called configurations of $\mathcal{B}$. These configurations are Euclidean metrics and the set $\operatorname{Conf} \mathcal{B}$ is subject to several conditions. In order to state these, one needs several concepts:

1) A class Fr of fit regions, which are subsets Euclidean spaces. These regions are those that a body system can occupy. Intuitively, the term "body" suggest that the regions it can occupy are connected. We do not assume this but, for simplicity, we will use the term "body" rather than "body system" from now on.

2) A class $\mathrm{Pl} \mathcal{B}$ of placements of the body, which are invertible mappings from the body $\mathcal{B}$ to a fit region in some Euclidean space, called the framespace of the placement.

3) A class $\operatorname{Tr}$ of transplacements, which are invertible mappings from a fit region in some framespace to a fit region in the same or another framespace.

The precise definitions and conditions that these concept must satisfy are, to some extent, quite technical and can be found in [NS1].

Each configuration $\delta \in \operatorname{Conf} \mathcal{B}$ can be used to construct a Euclidean space $\mathcal{E}_{\delta}$ and and an imbedding $\operatorname{imb}_{\delta}$, which is a special placement of the body in the frame-space $\mathcal{E}_{\delta}$. These special frame-spaces are internal, because they constructed from configurations; they are not external, i.e., introduced from the 
outside. In general, placements are in frame-spaces introduced from the outside.

Every placement $\mu$ induces a configuration $\delta_{\mu}$, but infinitely many placements all induce the same configuration.

Given a continuous body $\mathcal{B}$, consider the set $\Omega_{\mathcal{B}}$ defined by

$$
\begin{aligned}
& \Omega_{\mathcal{B}}:=\left\{\mathcal{P} \in \operatorname{SubB} \mid \operatorname{imb}_{\delta>}(\mathcal{P}) \in \operatorname{Fr}\right. \\
&\text { for every } \delta \in \operatorname{Conf} \mathcal{B}\} .
\end{aligned}
$$

In (3.6) "every" can be replaced by "some" without change of meaning.

The members of $\Omega_{\mathcal{B}}$ are called parts or subbodies of $\mathcal{B}$.

$\Omega_{\mathcal{B}}$ is materially ordered, as defined in Sect.3.1, by inclusion. It has the structure of a Boolean algebra with

$$
\begin{gathered}
\mathcal{P} \wedge \mathcal{Q}:=\mathcal{P} \cap \mathcal{Q}, \\
\mathcal{P} \vee \mathcal{Q}:=\operatorname{Int} \operatorname{Clo}(\mathcal{P} \cup \mathcal{Q}), \\
\mathcal{P}^{\text {rem }}:=\operatorname{Int}(\mathcal{B} \backslash \mathcal{P}),
\end{gathered}
$$

The proof of this highly non-trivial result can be found in $[\mathrm{NV}]$.

\subsection{Densities and Contactors}

We assume that a continuous body $\mathcal{B}$ is given. For each part $\mathcal{P} \in \Omega_{\mathcal{B}}$ and each placement $\mu \in \operatorname{Pl} \mathcal{B}$ we use the notation $\mathcal{P}_{\mu}:=\mu_{>}(\mathcal{P})$ and, in particular. $\mathcal{B}_{\mu}:=\operatorname{Rng} \mu$. We say that a part $\mathcal{P} \in \Omega_{\mathcal{B}}$ is internal if, for every placement $\mu \in \mathrm{Pl} \mathcal{B}$, we have Clo $\mathcal{P}_{\mu} \subset \mathcal{B}_{\mu}$. We denote the set of all internal parts by $\Omega_{\mathcal{B}}^{\text {int }}$.

Definition 4. An additive mapping $\mathbf{H}: \Omega_{\mathcal{B}} \longrightarrow \mathcal{W}$ is said to have densities if, for every $\mu \in \mathrm{Pl} \mathcal{B}$, there is a continuous mapping $\mathbf{h}_{\mu}: \mathcal{B}_{\mu} \longrightarrow \mathcal{W}$ such that

$$
\mathbf{H}(\mathcal{P})=\int_{\mathcal{P}_{\mu}} \mathbf{h}_{\mu} \quad \text { for all } \mathcal{P} \in \Omega_{\mathcal{B}}^{\text {int }}
$$

We call $\mathbf{h}_{\mu}$ the density of $\mathbf{H}$ in the placement $\mu$.

Definition 5. We say that an interaction $\mathbf{I}$ : $\left(\Omega_{\mathcal{B}}\right)_{\text {sep }}^{2} \longrightarrow \mathcal{W}$ has contactors if, for every placement $\mu$, there is a $C^{1}$ mapping $\mathbf{C}_{\mu}: \mathcal{B}_{\mu} \longrightarrow$ $\operatorname{Lin}\left(\mathcal{V}_{\mu}, \mathcal{W}\right)$ such that

$$
\mathbf{I}(\mathcal{P}, \mathcal{Q})=\int_{\operatorname{Rct}_{\mu}(\mathcal{P}, \mathcal{Q})} \mathbf{C}_{\mu} \mathbf{n}_{\mathcal{P}_{\mu}}
$$

for all $(\mathcal{P}, \mathcal{Q}) \in\left(\Omega_{\mathcal{B}}\right)_{\text {sep }}^{2}$ with $\mathcal{P} \in \Omega_{\mathcal{B}}^{\text {int }}$.

We call $\mathbf{C}_{\mu}$ the contactor of $\mathbf{I}$ in the placement $\mu$.

In both these definitions, "every" can be replaced by "some" without change of meaning.
Theorem 3: Given an interaction I : $\left(\Omega_{\mathcal{B}}\right)_{\text {sep }}^{2} \longrightarrow$ $\mathcal{W}$ with contactors and an additive mapping $\mathbf{H}: \Omega_{\mathcal{B}} \longrightarrow \mathcal{W}$ with densities, the following three conditions are equivalent:

1) We have

$$
\operatorname{Res}_{\mathbf{I}}(\mathcal{P})+\mathbf{H}(\mathcal{P})=\mathbf{0} \quad \text { for all } \mathcal{P} \in \boldsymbol{\Omega}_{\mathcal{B}}^{\text {int }} .
$$

2) For every placement $\mu \in \operatorname{Pl} \mathcal{B}$, we have

$$
\operatorname{div} \mathbf{C}_{\mu}+\mathbf{h}_{\mu}=\mathbf{0} \text {. }
$$

where $\mathbf{h}_{\mu}$ is the density of $\mathbf{H}$ in the placement $\mu$, and $\mathbf{C}_{\mu}$ is the contactor of $\mathbf{I}$ in the placement $\mu$.

3) Condition 2) holds with with "every" replaced by "some".

\subsection{Balance of Forces and Torques}

It is often useful to fix a Euclidean space $\mathcal{E}$, with translation space $\mathcal{V}$, and confine one's attention to placements whose range space is $\mathcal{E}$. It is then useful to consider force systems with values in $\mathcal{V}$, independent of the choice of a configuration, as follows:

Definition 6. A force system in the space $\mathcal{V}$ is a pair $\left(\mathbf{F}^{\mathrm{i}}, \mathbf{F}^{\mathrm{e}}\right)$, where $\mathbf{F}^{\mathrm{i}}:\left(\Omega_{\mathcal{B}}\right)_{\text {sep }}^{2} \longrightarrow \mathcal{V}$ is an interaction and $\mathbf{F}^{\mathrm{e}}: \Omega_{\mathcal{B}} \longrightarrow \mathcal{V}$ is additive. The mapping $\mathbf{F}^{\mathrm{i}}$ is called the internal force system in $\mathcal{V}$ and $\mathbf{F}^{\mathrm{e}}$ is called the external force system in $\mathcal{V}$.

Let a force system $\left(\mathbf{F}^{\mathrm{i}}, \mathbf{F}^{\mathrm{e}}\right)$ in $\mathcal{V}$ be a given. The first fundamental law of mechanics, called the Balance of Forces, says:

$$
\operatorname{Res}_{\mathbf{F}^{\mathrm{i}}}(\mathcal{P})+\mathbf{F}^{\mathrm{e}}(\mathcal{P})=\mathbf{0} \text { for all } \mathcal{P} \in \Omega_{\mathcal{B}} .
$$

We say that the system $\left(\mathbf{F}^{\mathrm{i}}, \mathbf{F}^{\mathrm{e}}\right)$ is forcebalanced if (14) holds.

Since $\mathbf{F}^{\mathrm{e}}$ is additive, the following Law of Action and Reaction is an immediate consequence of (14) and Theorem 2:

Every balanced balanced internal force system is skew, i. e. ,

$$
\mathbf{F}^{\mathrm{i}}(\mathcal{P}, \mathcal{Q})=-\mathbf{F}^{\mathrm{i}}(\mathcal{Q}, \mathcal{P}) \quad \text { for all } \quad(\mathcal{P}, \mathcal{Q}) \in\left(\Omega_{\mathcal{B}}\right)_{\text {sep }}^{2} .
$$

Remark 2: The law of action and reaction is often referred to as Newton's Third Law. Thus, if one assumes the balance law (3.14), one can prove Newton's Third Law instead of assuming it a priori, as Newton and many physics textbooks since Newton have done. The balance of forces has been understood by engineers, if only implicitly, since antiquity.

We now assume now that $\mathbf{F}^{\mathrm{i}}$ has contactors and $\mathbf{F}^{\mathrm{e}}$ has densities..

Let $\mu$ be a placement of the body in $\mathcal{E}$ and put $\mathcal{B}_{\mu}:=\mu_{>}(\mathcal{B})$. Let $\mathbf{T}_{\mu}: \mathcal{B}_{\mu}: \longrightarrow \operatorname{Lin} \mathcal{V}$ denote the contactor for $\mathbf{F}^{\mathrm{i}}$ and let $\mathbf{b}_{\mu}: \mathcal{B}_{\mu}: \longrightarrow \mathcal{V}$ denote the 
density of $\mathbf{F}^{\mathrm{e}}$ in the given the placement $\mu$. It follows from Theorem 3 that the force balance, restricted to internal parts $\mathcal{P}$, is equivalent to

$$
\operatorname{div} \mathbf{T}_{\mu}+\mathbf{b}_{\mu}=\mathbf{0}
$$

It is usual to assume that the force system is not only force-balanced but also torque-balanced. We will not give a precise definition here but note that the condition

$$
\operatorname{Rng} \mathbf{T}_{\mu} \subset \operatorname{Sym} \mathcal{V}
$$

is equivalent to balance of torques for internal parts.

From here on we will assume that (16) and (17) are valid. We adjust the codomain of $\mathbf{T}_{\mu}$ to Sym $\mathcal{V}$ without change of notation and call $\mathbf{T}_{\mu}: \mathcal{B}_{\mu}: \longrightarrow$ Sym $\mathcal{V}$ the Cauchy stress of the force system in the placement $\mu$ and the mapping $\mathbf{b}_{\mu}: \mathcal{B}_{\mu} \longrightarrow \mathcal{V}$ the external body-force density in the placement $\mu$.

Let a configuration $\delta \in \operatorname{Conf} \mathcal{B}$ be given. As in Section 3.4, we denote the imbedding space for $\delta$ by $\mathcal{E}_{\delta}$ and its translation space by $\mathcal{V}_{\delta}$, and we use the results above in the case when $\mu:=\operatorname{imb}_{\delta}$ and write, for simplicity, $\delta$ rather than $\operatorname{imb}_{\delta}$ as a subscript.

Definition 7. $A$ force system in the configuration $\delta$ is a pair $\left(\mathbf{F}_{\delta}^{\mathrm{i}}, \mathbf{F}_{\delta}^{\mathrm{e}}\right)$ which is a force system, both force-balanced and torque-balanced, in the space $\mathcal{V}_{\delta}$ in the sense of Definition 6.

Let such a force system $\left(\mathbf{F}_{\delta}^{\mathrm{i}}, \mathbf{F}_{\delta}^{\mathrm{e}}\right)$ in $\mathcal{V}_{\delta}$ be given. We assume that $\mathbf{F}_{\delta}^{\mathrm{i}}$ has contactors and that $\mathbf{F}_{\delta}^{\mathrm{e}}$ has densities. The results (3.16), and (17) remain valid when the subscript $\delta$ is used instead of $\mu$, when $\mathbf{T}_{\delta}$ is interpreted to be the contactor of $\mathbf{F}_{\delta}^{\mathrm{i}}$ in the placement $\mathrm{imb}_{\delta}$, and when $\mathbf{b}_{\delta}$ is interpreted to be the density of $\mathbf{F}_{\delta}^{\mathrm{e}}$ in the placement $\mathrm{imb}_{\delta}$. We may call $\mathbf{T}_{\delta}$ the configurational stress and $i m b_{\delta}$ the configurational body force density for $\delta$.

Definition 8. A mechanical process is a timefamily $\left(\left(\bar{\delta}_{t}, \overline{\mathbf{F}}_{t}^{\mathrm{i}}, \overline{\mathbf{F}}_{t}^{\mathrm{e}}\right) \mid t \in I\right)$ of triples, where $\left(\bar{\delta}_{t} \mid t \in I\right)$ is a deformation process and, for every $t \in I,\left(\overline{\mathbf{F}}_{t}^{\mathrm{i}}, \overline{\mathbf{F}}_{t}^{\mathrm{e}}\right)$ is a force system, both force-balanced and torquebalanced, in the configuration $\bar{\delta}_{t}$, as defined by Definition 7 .

\subsection{Energy Balance}

Energy and heat are fundamental concepts in thermodynamics.

Definition 9. A heat transfer system is a pair $\left(Q^{\mathrm{i}}, Q^{\mathrm{e}}\right)$, where $Q^{\mathrm{i}}:\left(\Omega_{\mathcal{B}}\right)_{\text {sep }}^{2} \longrightarrow \mathbb{P}^{\times}$is an interaction and $Q^{\mathrm{e}}: \Omega_{\mathcal{B}} \longrightarrow \mathbb{P}^{\times}$is additive. The mapping $Q^{\mathrm{i}}$ is called the internal heat transfer and $Q^{\mathrm{e}}$ is called the external heat absorption.
We assume that $Q^{\mathrm{e}}$ has densities and $Q^{\mathrm{i}}$ has contactors. Hence, given any placement $\mu$ in a framespace $\mathcal{E}$ with translation space $\mathcal{V}$, we obtain a density $r_{\mu}: \mathcal{B}_{\mu} \longrightarrow \mathbb{R}$ and a contactor $-\mathbf{q}_{\mu}: \mathcal{B}_{\mu} \longrightarrow$ $\operatorname{Lin}(\mathcal{V} \cdot \mathbb{R}) \cong \mathcal{V}$ such that

$$
\begin{array}{r}
Q^{\mathrm{e}}(\mathcal{P})=\int_{\mathcal{P}_{\mu}} r_{\mu} \quad \text { for all } \mathcal{P} \in \Omega_{\mathcal{B}}^{\mathrm{int}} . \\
Q^{\mathrm{i}}(\mathcal{P}, \mathcal{Q})=-\int_{\operatorname{Rct}_{\mu}(\mathcal{P}, \mathcal{Q})} \mathbf{q}_{\mu} \cdot \mathbf{n}_{\mathcal{P}_{\mu}}
\end{array}
$$

for all $(\mathcal{P}, \mathcal{Q}) \in\left(\Omega_{\mathcal{B}}\right)_{\text {sep }}^{2}$ with $\mathcal{P} \in \Omega_{\mathcal{B}}^{\text {int }}$.

$r_{\mu}$ is called the heat absorption field and $\mathbf{q}_{\mu}$ the heat flux of the given heat transfer system in the placement $\mu$.

Definition 10 An energetic process is a sextuple $\left(\left(\bar{\delta}_{t}, \overline{\mathbf{F}}_{t}^{\mathrm{i}}, \overline{\mathbf{F}}_{t}^{\mathrm{e}}, \bar{Q}_{t}^{\mathrm{i}}, \bar{Q}_{t}^{\mathrm{e}}, \bar{E}_{t}\right) \mid t \in I\right)$ of time-families such that $\left(\left(\bar{\delta}_{t}, \overline{\mathbf{F}}_{t}^{\mathrm{i}}, \overline{\mathbf{F}}_{t}^{\mathrm{e}}\right) \mid t \in I\right)$ is a mechanical process, $\left(\left(\bar{Q}_{t}^{\mathrm{i}}, \bar{Q}_{t}^{\mathrm{e}}\right) \mid t \in I\right)$ is a time-family of heat transfer systems, and $\left(\bar{E}_{t} \mid t \in I\right)$ is an appropiatly defined differentiable time-family of additive mappings, called the internal energy.

We say that a given energetic process $\left(\left(\bar{\delta}_{t}, \overline{\mathbf{F}}_{t}^{\mathrm{i}}, \overline{\mathbf{F}}_{t}^{\mathrm{e}}, \bar{Q}_{t}^{\mathrm{i}}, \bar{Q}_{t}^{\mathrm{e}}, \bar{E}_{t}\right) \mid t \in I\right)$ is energy-balanced if

$$
\begin{gathered}
\bar{P}_{t}(\mathcal{P})+\operatorname{Res}_{\bar{Q}_{t}^{\mathrm{i}}}(\mathcal{P})+\bar{Q}_{t}^{\mathrm{e}}(\mathcal{P})=\bar{E}_{t}^{\bullet}(\mathcal{P}) \\
\text { for all } \mathcal{P} \in \Omega_{\mathcal{B}} \text { and } t \in I,
\end{gathered}
$$

where $\left(\bar{P}_{t} \mid t \in I\right)$ is the power-family determined by the mechanical process $\left(\left(\bar{\delta}_{t}, \overline{\mathbf{F}}_{t}^{\mathrm{i}}, \overline{\mathbf{F}}_{t}^{\mathrm{e}}\right) \mid t \in I\right)$.

Under suitable conditions, the energy balance law can be given a local form

\subsection{Temperature and Entropy}

Theorem 6. Let a heat transfer system $\left(Q^{\mathrm{i}}, Q^{\mathrm{e}}\right)$, as defined by Definition 10, and a function $\theta: \mathcal{B} \longrightarrow \mathbb{P}^{\times}$ of class $C^{1}$, called temperature, be given, and assume that $Q^{\mathrm{i}}$ has contactors and $Q^{\mathrm{e}}$ has densities. Then there is a pair $\left(H^{\mathrm{i}}, H^{\mathrm{e}}\right)$, where $H^{\mathrm{i}}:\left(\Omega_{\mathcal{B}}\right)_{\text {sep }}^{2} \longrightarrow$ $\mathbb{R}$ is an interaction and $H^{\mathrm{e}}: \Omega_{\mathcal{B}} \longrightarrow \mathbb{R}$ is the additive function such that, for every placement $\mu$ we have

$$
H^{\mathrm{i}}(\mathcal{P}, \mathcal{Q})=-\int_{\operatorname{Rct}_{\mu}(\mathcal{P}, \mathcal{Q})} \frac{\mathbf{q}_{\mu}}{\theta_{\mu}} \cdot \mathbf{n}_{\mathcal{P}_{\mu}}
$$

$$
\text { for all }(\mathcal{P}, \mathcal{Q}) \in\left(\Omega_{\mathcal{B}}\right)_{\text {sep }}^{2} \text { with } \mathcal{P} \in \Omega_{\mathcal{B}}^{\text {int }}
$$

and

$$
H^{\mathrm{e}}(\mathcal{P})=\int_{\mathcal{P}_{\mu}} \frac{r_{\mu}}{\theta_{\mu}} \quad \text { for all } \mathcal{P} \in \Omega_{\mathcal{B}}^{\text {int }}
$$

where $\mathbf{q}_{\mu}$ is the heat flux in the placement $\mu, r_{\mu}$ is the external heat absorption density in $\mu$ and $\theta_{\mu}:=\theta \circ \mu^{\leftarrow}$. The pair $\left(H^{\mathrm{i}}, H^{\mathrm{e}}\right)$ is called the entropy 
transfer system generated by the heat transfer system $\left(Q^{\mathrm{i}}, Q^{\mathrm{e}}\right)$ and the temperature $\theta . H^{\mathrm{i}}$ is called the internal entropy transfer, and $H^{\mathrm{e}}$ is called the external entropy absorption.

The proof is not very difficult.

Definition 11. Let by a time-famiy $\left(\left(\bar{Q}_{t}^{\mathrm{i}}, \bar{Q}_{t}^{\mathrm{e}}\right) \mid t \in\right.$ $I)$ of heat transfer systems and a time-family of temperatures $\left(\bar{\theta}_{t} \mid t \in I\right)$ be given, and let $\left(\left(\bar{H}_{t}^{\mathrm{i}}, \bar{H}_{t}^{\mathrm{e}}\right) \mid t \in\right.$ $I)$ be the resulting entropy transfer system as described in Theorem 6. Let $\left(\bar{N}_{t}: \Omega_{\mathcal{B}} \longrightarrow \mathbb{R} \mid t \in I\right)$ be a differentiable time-family of additive mappings, called the internal entropy.

We say that $\left(\left(\bar{H}_{t}^{\mathrm{i}}, \bar{H}_{t}^{\mathrm{e}}, \bar{N}_{t}\right) \mid t \in I\right)$ is a dissipative entropical process if

$$
\begin{gathered}
\bar{N}_{t}^{\bullet}(\mathcal{P}) \geq \operatorname{Res}_{\bar{H}_{t}^{\mathrm{i}}}(\mathcal{P})+\bar{H}_{t}^{\mathrm{i}}(\mathcal{P}) \\
\text { for all } \mathcal{P} \in \Omega_{\mathcal{B}}^{\mathrm{int}} \text { and } t \in I .
\end{gathered}
$$

Under suitable conditions, the energy balance law can be given a local form.

Using the local form of the energy balance law and the local form of the dissipation inequality, one can obtain the following intrinsic reduced dissipation inequality:

$$
\bar{\rho}_{\mathrm{m}}\left(\bar{\theta} \bar{\eta}^{\bullet}-\bar{\epsilon}^{\bullet}\right)+\frac{1}{2} \operatorname{tr}\left(\overline{\mathbf{S}} \overline{\mathbf{G}}^{\bullet}\right)-\frac{1}{\bar{\theta}} \bar{\gamma} \overline{\mathbf{h}} \geq 0,
$$

where $\bar{\gamma}(X, t):=\nabla_{X} \bar{\theta}(\cdot, t) \in \mathcal{T}_{X}^{*} \quad$ for all $\quad X \in \mathcal{B}$, $t \in I$, and where the intrinsic stress $\overline{\mathbf{S}}$ and the intrinsic heat flux $\overline{\mathbf{h}}$ are related to the Cauchy-stress $\overline{\mathbf{T}}$ and the heat flux $\overline{\mathbf{q}}$ by

$$
\overline{\mathbf{M}} \overline{\mathbf{S}} \overline{\mathbf{M}}^{\top}=\overline{\mathbf{T}}_{\mathrm{m}}, \quad \overline{\mathbf{M}} \mathbf{h}=\overline{\mathbf{q}}_{\mathrm{m}},
$$

respectively, with $\overline{\mathbf{M}}(X, t):=\nabla_{X} \mu_{t} \in \operatorname{Lis}\left(\mathcal{T}_{X}, \mathcal{V}\right)$ for all $X \in \mathcal{B}, t \in I$.

The inequality (24) involves only the (internal) thermomechanical process

$$
\left(\bar{\delta}, \bar{\theta}, \overline{\mathbf{F}}^{\mathrm{i}}, \bar{Q}^{\mathrm{i}}, \bar{N}, \bar{E}\right)
$$

and not the external forces, nor the external heat transfer, nor any external frame of reference. It is (24) that is used to determine restrictions on framefree formulated constitutive laws, as will be explained in a later presentation by Brian Seguin.

\section{References:}

[NS1] W. Noll and B. Seguin: Basic Concepts of Thermomechanics, 20 pages (2009). Published as B5 on the website www.math.cmu.edu/ wnog/noll .

[NLFT] C. Truesdell and W. Noll: The NonLinear Field Theories of Mechanics, Encyclopedia of Physics, Vol. III/3, 602 pages. Springer-Verlag, 1965. Second Edition, 1992. Translation into Chinese, 2000. Third Edition, 2004.

[F] R. P. Feynmann, R.B. Leighton. and M. Sands, The Feynmann Lectures on Physics, Vol. I, AddisonWesley, Reading 1963.

[C] B. D. Coleman: Memories of Clifford Truesdell, Journal of Elasticity 70, 1-13 (2003).

[CN] B. D. Coleman and W. Noll: The Thermodynamics of Elastic Materials with Heat Conduction and Viscosity, Archive for Rational Mechanics and Analysis 13, 167-178 (1964).

[N1] W. Noll: A new mathematical theory of simple materials, Archive for Rational Mechanics and Analysis 48, 1-50 (1972).

[FC]: W. Noll: Five Contributions to Natural Philosophy, 73 pages (2004). Published as B1 on the website www.math.cmu.edu/ wnog/noll .

[N2] W. Noll: The Conceptual Infrastructure of Mathematics, 5 pages (1995). Published as A2 on the website www.math.cmu.edu/ wnog/noll .

[N3] W. Noll: A Frame-Free Formulation of Elasticity. Journal of Elasticity 83, 291-307 (2006). Also published as B4 on the website www.math.cmu.edu/ wnog/noll.

[N4] W. Noll: Proof of the maximality of the orthogonal group in the unimodular group, Archive for Rational Mechanics and Analysis 18, 100-102 (1965).

[FDS] Noll, W. :Finite-Dimensional Spaces: Algebra, Geometry, and Analysis, Vol.I and Vol.II. Published as $\mathrm{C} 1$ and $\mathrm{C} 2$ on the website www. math.cmu.edu/ wnog/noll. (Vol.I was published by Martinus Nijhoff Publishers in 1987.) Vol.I has 393 pages. The preliminary manuscript of Vol.II has about 110 pages and is growing.

[NS2] W. Noll and B. Seguin: Monoids, Boolean Algebras, Materially Ordered Sets, International Journal of Pure and Applied Mathematics 37, 187202 (2007). Also published as C4 on the website www.math.cmu.edu/ wnog/noll.

[NV] W,Noll and E.Virga: Fit regions and functions of bounded variation, Archive for Rational Mechanics and Analysis 102, 1-21 (1988), 